\title{
Albuminuria and Estimated Glomerular Filtration Rate Independently Associate with Acute Kidney Injury
}

\author{
Morgan E. Grams, ${ }^{\star}$ Brad C. Astor, ${ }^{{ }^{\dagger}}{ }^{\dagger}$ Lori D. Bash, ${ }^{\dagger}$ Kunihiro Matsushita, ${ }^{\dagger}$ Yaping Wang, ${ }^{\ddagger}$ \\ and Josef Coresh ${ }^{\star \ddagger}$ \\ *Department of Medicine, The Johns Hopkins University School of Medicine, Baltimore, Maryland; and \\ Departments of ${ }^{\dagger}$ Epidemiology and ${ }^{\ddagger}$ Biostatistics, The Johns Hopkins Bloomberg School of Public Health, \\ Baltimore, Maryland
}

\begin{abstract}
Acute kidney injury (AKI) is increasingly common and a significant contributor to excess death in hospitalized patients. CKD is an established risk factor for AKI; however, the independent graded association of urine albumin excretion with AKI is unknown. We analyzed a prospective cohort of 11,200 participants in the Atherosclerosis Risk in Communities (ARIC) study for the association between baseline urine albumin-to-creatinine ratio and estimated GFR (eGFR) with hospitalizations or death with AKI. The incidence of AKI events was 4.0 per 1000 person-years of follow-up. Using participants with urine albumin-to-creatinine ratios $<10 \mathrm{mg} / \mathrm{g}$ as a reference, the relative hazards of $\mathrm{AKI}$, adjusted for age, gender, race, cardiovascular risk factors, and categories of eGFR were $1.9(95 \% \mathrm{Cl}, 1.4$ to 2.6$), 2.2$ (95\% $\mathrm{Cl}, 1.6$ to 3.0$)$, and $4.8(95 \% \mathrm{Cl}, 3.2$ to 7.2$)$ for urine albumin-to-creatinine ratio groups of 11 to $29 \mathrm{mg} / \mathrm{g}$, 30 to $299 \mathrm{mg} / \mathrm{g}$, and $\geq 300 \mathrm{mg} / \mathrm{g}$, respectively. Similarly, the overall adjusted relative hazard of AKI increased with decreasing eGFR. Patterns persisted within subgroups of age, race, and gender. In summary, albuminuria and eGFR have strong, independent associations with incident AKI.
\end{abstract}

J Am Soc Nephrol 21: $\bullet \bullet-\bullet \bullet, ~ 2010$. doi: 10.1681/ASN.2010010128

It has long been recognized that an episode of acute kidney injury (AKI) can have serious health consequences. ${ }^{1-4}$ Even a relatively small degree of renal injury increases a patient's risk of a prolonged hospital stay, chronic kidney disease (CKD), ESRD, and death., ${ }^{2,5-10}$ Over the last 2 decades, the incidence of hospitalized AKI has increased dramatically. ${ }^{11-14}$ Precise estimations vary depending on population and method of case identification, but a recent community-based study of AKI estimated the incidence of nondialysis requiring AKI at 522 per 100,000 population per year and dialysis-requiring AKI at 30 per $100,000,{ }^{13}$ which is well over that of ESRD. ${ }^{14}$ This increase in the burden of disease, taken with the associated poor long-term outcomes, has established AKI as a major public health issue. ${ }^{14}$

Beyond routine supportive care, there exists little established medical therapy for AKI. ${ }^{15}$ Many current lines of research are focused on the prevention of AKI. However, few prospective, population-based studies have evaluated the development of AKI. ${ }^{3,13,16} \mathrm{Hsu}$ et al., ${ }^{13,17}$ along with multiple observational series in various clinical settings, have clearly established older age and CKD as risk factors for AKI. ${ }^{18-24}$ Other observed associations with AKI include black race and male gender. ${ }^{11,18,25}$ Proteinuria, an established risk factor in the development of cardiovascular disease, ${ }^{26,27}$ ESRD, ${ }^{28}$ and death, ${ }^{29}$ is less studied in its role in the development of AKI. Hsu and colleagues demonstrated the prospective association of proteinuria with dialysis-requiring AKI; however, the proteinuria clas-

Received January 30, 2010. Accepted April 20, 2010.

Published online ahead of print. Publication date available at www.jasn.org.

Correspondence: Dr. Josef Coresh, Director, Cardiovascular Epidemiology and Comstock Center, 2024 East Monument Street, Suite 2-600, Baltimore, MD 21287. Phone: 410-955-0495; Fax: 410-955-0476; E-mail: coresh@jhu.edu

Copyright (C) 2010 by the American Society of Nephrology 
sification was binary and based on dipstick measurement. ${ }^{17}$ To our knowledge, no study has quantified the independent dose response of albuminuria with AKI hospitalization, including less severe AKI. Our study's objective was thus to characterize prospectively the association between baseline urine albumin-to-creatinine ratio (UACR) and hospitalizations for AKI, controlling for established and potential risk factors such as $\mathrm{CKD}$, age, and cardiovascular comorbidities.

\section{RESULTS}

\section{AKI Incidence}

Among 11,200 cohort participants with nonmissing baseline data, the average follow-up was 8.0 years. There were 17,265 hospitalizations during the follow-up period, including 492 (2.8\% of the total) with an International Classification of Diseases (ICD) code indicating AKI. These 492 AKI hospitalizations occurred among 356 participants. Most events were captured from the discharge diagnosis ICD code; the associated death certificate solely identified $<1 \%$ percent of AKI hospitalizations. Using only a participant's first AKI event, the incidence rate of AKI was 4.0 events per 1000 person-years. This rate increased significantly over time. Over the first 4 years after baseline visit, the incidence of AKI was 2.2 events per 1000 person-years (95\% confidence interval [CI] 1.8 to 2.6 ) compared with 5.7 events per 1000 person-years ( $95 \%$ CI, 5.1 to 6.5) in the subsequent years of follow-up. The overall incidence of hospitalization with AKI as the first discharge diagnosis (and thus possibly a hospitalization for AKI) was 0.8 events per 1000 person-years. The mortality rate in the cohort was 13.2 deaths per 1000 person-years.

\section{Characteristics of Participants}

Baseline characteristics of participants with an AKI event were markedly different from those without an AKI event (Table 1). The AKI group was slightly older at baseline, with a higher percentage of men and black participants. They were more likely to have diabetes, hypertension, obesity, cardiovascular disease, CKD, and albuminuria than their counterparts without an AKI event. A significantly greater proportion of the AKI group used an angiotensin-converting enzyme inhibitor (ACEI) or angiotensin II receptor blocker (ARB) at baseline visit. The mean estimated GFR (eGFR) was lower in the AKI group, irrespective of whether the Modification of Diet in Renal Disease (MDRD) or CKD-EPI was used as the method of estimation. The proportion of smokers was not significantly different. The proportion with a known previous AKI hospitalization also did not significantly differ between the groups, although only 20 participants fit this category.

During the follow-up period, individuals with an AKI hospitalization had more hospitalizations for any cause (6.0 hospitalizations over 7.0 years) than their counterparts without an AKI hospitalization (1.3 hospitalizations over 8.1 years). They also were less likely to survive the follow-up period, with over half of those participants with at least one hospital discharge
Table 1. Baseline characteristics among ARIC participants stratified by any occurrence of AKI hospitalization ${ }^{a}$

\begin{tabular}{|c|c|c|}
\hline \multirow[b]{2}{*}{ Characteristics } & \multicolumn{2}{|c|}{ Total Cohort } \\
\hline & $\begin{array}{c}\text { No AKI } \\
\text { Hospitalization }\end{array}$ & $\begin{array}{c}\text { AKI } \\
\text { Hospitalization }\end{array}$ \\
\hline$n$ & 10844 & 356 \\
\hline Percent & $96.8 \%$ & $3.2 \%$ \\
\hline Age (mean) & 62.8 & 64.7 \\
\hline Female (\%) & 56.0 & 48.3 \\
\hline Race (\% black) & 21.7 & 34.6 \\
\hline Diabetes (\%) & 16.0 & 39.9 \\
\hline Hypertension (\%) & 46.7 & 70.4 \\
\hline Obese (\%) & 34.6 & 48.7 \\
\hline Current smoking (\%) & 14.7 & 18.2 \\
\hline Baseline ACEI/ARB (\%) & 12.1 & 27.5 \\
\hline Prior cardiovascular event (\%) & 8.1 & 24.7 \\
\hline Prior $\mathrm{AKI}$ event $(\%)^{\mathrm{b}}$ & 0.2 & 0.6 \\
\hline MDRD GFR (median) & 81.0 & 72.2 \\
\hline CKD-EPI GFR (median) & 86.4 & 75.4 \\
\hline Stage 3 to 4 CKD by MDRD (\%) & 7.6 & 27.5 \\
\hline $\begin{array}{l}\text { Stage } 3 \text { to } 4 \text { CKD by } \\
\text { CKD-EPI (\%) }\end{array}$ & 5.9 & 25.0 \\
\hline UACR (mean) & 23.2 & 207.1 \\
\hline Microalbuminuria (\%) & 6.2 & 16.9 \\
\hline Macroalbuminuria (\%) & 1.3 & 13.2 \\
\hline Mean follow-up, years & 8.1 & 7.0 \\
\hline Number of admissions, mean & 1.3 & 6.0 \\
\hline Deaths (\%) & 9.3 & 52.8 \\
\hline
\end{tabular}

${ }^{a}$ All $P$ values $<0.001$ with the exception of $\%$ female $(P=0.004)$, \% current smoking $(P=0.1)$, \% prior known AKI event $(P=0.1)$

brior AKI event defined as an AKI hospitalization since enrollment in the ARIC study but before study visit 4 .

code for AKI dying during follow-up, compared with $9.3 \%$ in the non-AKI group.

\section{Characteristics of Hospitalizations}

Hospitalizations with a discharge diagnosis code for AKI were quite different from hospitalizations without such a code (Table 2). Overall, hospitalizations with AKI made up 2.8\% of the 17,265 cohort hospitalizations. Mean length of stay for an AKI hospitalization was longer, at 14.6 days versus 5.5 days. On average, AKI hospitalizations happened later during the follow-up period than non-AKI hospitalizations ( 5.6 years versus 4.5 years).

There was no significant difference in proportion of hospitalizations for open heart surgery or percutaneous coronary intervention ("high-risk" hospitalizations) between the nonAKI and AKI hospitalizations. Nearly one-quarter of the AKI hospitalizations listed AKI as the first discharge diagnosis, and $12.8 \%$ required dialysis during the hospital stay. In-hospital mortality was much higher during AKI hospitalizations than non-AKI hospitalizations, at $18.9 \%$ versus $2.5 \%$. For the subgroup of hospitalizations with AKI listed as the first discharge diagnosis, the proportion receiving dialysis was $23.2 \%$. Compared with other AKI hospitalizations, those with AKI as the first discharge diagnosis had a shorter average length of stay and a lower in-hospital mortality rate at $4.5 \%$. 
Table 2. Characteristics of hospitalizations, for non-AKI hospitalizations, all AKI hospitalizations, and hospitalizations with AKI listed as the first discharge diagnosis ${ }^{a}$

\begin{tabular}{|c|c|c|c|}
\hline \multirow[b]{2}{*}{ Total Cohort } & \multirow[b]{2}{*}{$\begin{array}{c}\text { Non-AKI } \\
\text { Hospitalizations }\end{array}$} & \multicolumn{2}{|c|}{ AKI by ICD Code in } \\
\hline & & $\begin{array}{c}\text { Any } \\
\text { Position } \\
\text { (all AKI) }\end{array}$ & $\begin{array}{c}\text { First } \\
\text { Position } \\
\text { (for AKI) }\end{array}$ \\
\hline$n$ & 16,773 & 492 & 112 \\
\hline Percent of total hospitalizations & $97.2 \%$ & $2.8 \%$ & $0.6 \%$ \\
\hline Mean length of stay, days & 5.5 & 14.6 & 9.7 \\
\hline Mean time since visit 4, days & 1647.7 & 2047.1 & 2096.6 \\
\hline High-risk hospitalization (\%) & 5.4 & 5.7 & 0.0 \\
\hline AKI first diagnosis code (\%) & 0.0 & 22.8 & 100.0 \\
\hline Received dialysis in hospital (\%) & 1.9 & 12.8 & 23.2 \\
\hline In-hospital death (\%) & 2.5 & 18.9 & 4.5 \\
\hline
\end{tabular}

a For the comparison of non-AKI hospitalizations to AKI hospitalizations, all $P$ values $<0.001$ with the exception of \% high-risk hospitalization $(P=0.8)$. For the comparison of $A K I$ hospitalizations defined by ICD code in the first position to those defined by an ICD in any other position (not shown), all $P$ values $<0.001$ with the exception of mean length of stay $(P=0.04)$ and mean time since visit $4(P=0.8)$.

bHigh-risk hospitalization defined as a hospitalization with an ICD-9 code for open heart surgery (including coronary bypass surgery) or percutaneous coronary intervention.

\section{AKI Incidence Stratified by Category of Albuminuria}

The crude risk of AKI was greater with greater levels of albuminuria. When stratified into groups of UACR $\leq 10 \mathrm{mg} / \mathrm{g}$ (no albuminuria), 10 to $29 \mathrm{mg} / \mathrm{g}$ (subclinical albuminuria), 30 to $299 \mathrm{mg} / \mathrm{g}$ (microalbuminuria), and $\geq 300 \mathrm{mg} / \mathrm{g}$ (macroalbuminuria), there was a stepwise increase in incidence of AKI, from 2.6 events in the no albuminuria group, to 6.0 events in the subclinical albuminuria group, to 11.1 and 41.2 events per 1000 person years in the micro- and macroalbuminuria groups, respectively. As shown in Table 3, this effect persisted after stratification by eGFR; except within stage 4 (which encompassed only 25 participants), the incidence rate of AKI was consistently higher at higher levels of albuminuria. The trend was consistent when stratified by subgroups of age, gender, race, and presence of CKD (Figure 1).

\section{Adjusted Association between Baseline Renal Disease and AKI Hospitalization}

Table 4 demonstrates the stepwise increase in adjusted hazard ratio of AKI by level of albuminuria overall and within each category of eGFR. After adjustment for categories of eGFR, the adjusted hazard ratio was $1.9(95 \% \mathrm{CI}, 1.4$ to 2.6$), 2.2(95 \% \mathrm{CI}$,
1.6 to 3.0 ), and 4.8 (95\% CI, 3.2 to 7.2 ) in participants with subclinical, micro-, and macroalbuminuria, respectively, using participants with no albuminuria as a reference. This association persisted within age ( $<65$ years and $\geq 65$ years), gender, and race subgroups and when evaluating the risk of hospitalization with $\mathrm{AKI}$ as the first discharge diagnosis (results not shown).

Associations between continuous UACR and AKI incidence were consistent with the results of the stratified UACR (Figure 2). Adjusting for continuous eGFR, demographics, and cardiovascular risk factors and using a reference UACR of $5 \mathrm{mg} / \mathrm{g}$ and knots at 10,30 , and $300 \mathrm{mg} / \mathrm{g}$, a spline approach to model the relationship between UACR and adjusted hazard ratios for AKI resulted in a nearly linear relationship.

Overall, there was a graded association in the adjusted hazard ratio of AKI hospitalization with worsened eGFR (Table 4). After adjustment for categories of UACR, there was no significant difference between eGFR $>105 \mathrm{ml} / \mathrm{min}$ per $1.73 \mathrm{~m}^{2}$, eGFR between 90 and $104 \mathrm{ml} / \mathrm{min}$ per $1.73 \mathrm{~m}^{2}$ (the reference group), and eGFR between 75 and $89 \mathrm{ml} / \mathrm{min}$ per $1.73 \mathrm{~m}^{2}$; thereafter, $15 \mathrm{ml} / \mathrm{min}$ per $1.73 \mathrm{~m}^{2}$ decrements in eGFR were associated with adjusted hazard ratios of 1.5 (95\% CI, 1.1 to 2.1 ), 2.5 (95\% CI, 1.7 to 3.7), 7.0 (95\% CI, 4.4 to 11.0 ), and 5.6 (95\% CI, 2.6 to 12.1 ). This association was also consistent when eGFR was analyzed as a continuous variable. Figure 3 demonstrates the relationship between eGFR and adjusted risk of AKI using a linear spline approach.

\section{Sensitivity Analysis}

To take into account the possible confounding by high-risk medications and procedures on the association between baseline renal function and the risk of AKI, two separate analyses were performed. First, all multivariate analyses were repeated adjusting for baseline ACEI and ARB use. Although baseline ACE-I or ARB therapy was significantly associated with AKI (adjusted hazard ratio, $1.42,95 \%$ CI, 1.10 to 1.83 ), the adjusted hazard ratios were

Table 3. Incidence rate of AKI per 1000 person-years and total number of participants among categories of UACR and eGFR

\begin{tabular}{|c|c|c|c|c|c|}
\hline \multirow{2}{*}{ eGFR $\left(\mathrm{ml} / \mathrm{min}\right.$ per $\left.1.73 \mathrm{~m}^{2}\right)$} & \multicolumn{5}{|c|}{ Albumin:Creatinine Ratio $(\mathrm{mg} / \mathrm{g})$} \\
\hline & $<10$ & 10 to 29 & 30 to 299 & $\geq 300$ & All \\
\hline$\geq 105$ & $2.9(955)$ & $2.5(201)$ & $8.1(117)$ & $37.8(21)$ & 3.7 (1294) \\
\hline 75 to 89 & $1.7(2822)$ & $4.7(379)$ & $7.4(158)$ & $20.4(28)$ & $2.4(3387)$ \\
\hline 60 to 74 & $2.7(2427)$ & $8.2(328)$ & $8.7(166)$ & $41.6(43)$ & $4.0(2964)$ \\
\hline 45 to 59 & $5.7(535)$ & $14.6(96)$ & $23.1(105)$ & $26.2(25)$ & $9.5(761)$ \\
\hline All & $2.6(8931)$ & $6.0(1346)$ & $11.1(735)$ & $41.2(188)$ & $4.0(11200)$ \\
\hline
\end{tabular}




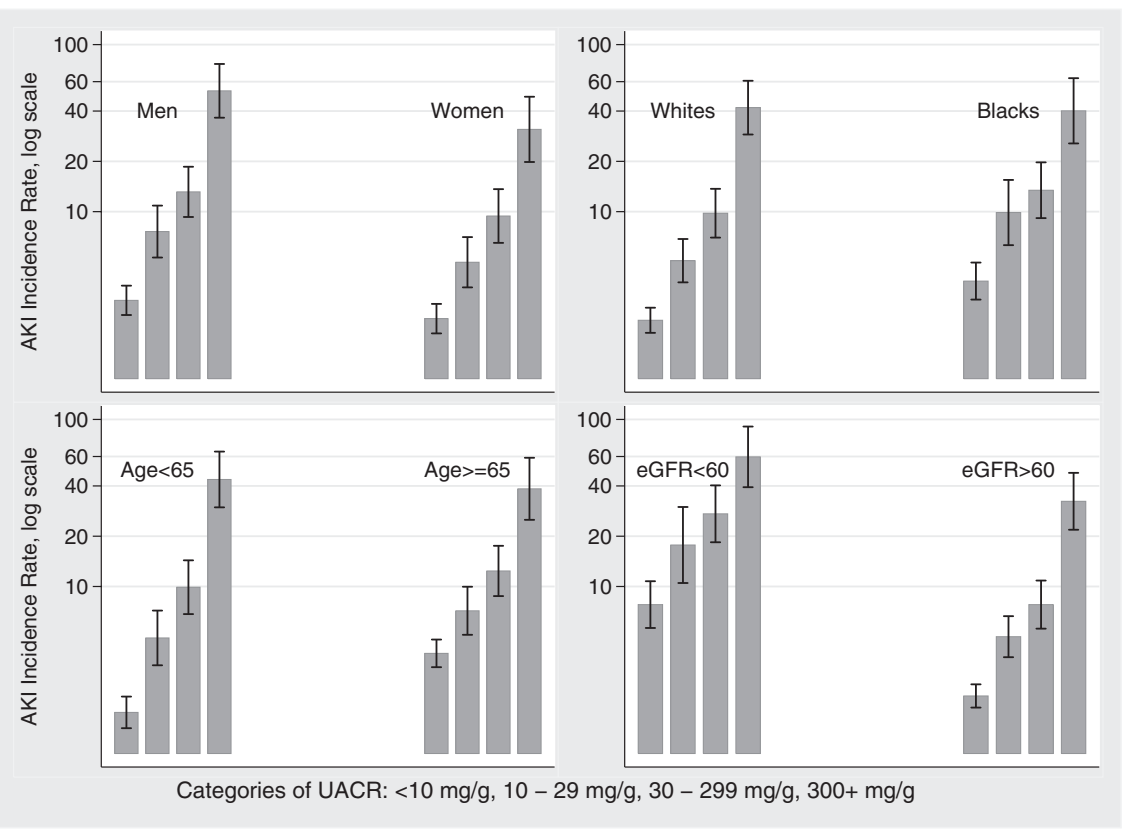

Figure 1. AKI incidence per 1000 person-years (with $95 \% \mathrm{Cl}$ ) increases with increasing $\operatorname{UACR}(<10,10$ to 29,30 to 299 , and $\geq 300 \mathrm{mg} / \mathrm{g}$ ) by subgroups of gender, race, age, and presence of CKD.

essentially unchanged within strata of UACR and eGFR. Second, analyses were repeated modeling AKI occurring only during non-high-risk hospitalizations. In this approach, the 25 AKI events that occurred during hospitalizations for open heart surgery or percutaneous coronary intervention were censored. Again, the hazard ratios of AKI associated with baseline UACR and eGFR were largely unchanged. Using participants with no albuminuria as the reference, the fully adjusted hazard ratio of AKI in a non-high-risk hospitalization was 1.9 (95\% CI, 1.4 to 2.6 ), 2.3 (95\% CI, 1.6 to 3.2 ), and 5.0 (95\% CI, 3.3 to 7.5 ) in participants with subclinical, micro-, and macroalbuminuria, respectively.

To determine the effect of time on the association between renal function and AKI risk, adjusted hazard ratios over the full follow-up period were compared with those estimated during the first 4 years after baseline visit. The adjusted hazard for AKI was generally higher by strata of UACR and eGFR in the early period, ranging from 2.6 (95\% CI, 1.4 to 4.8$)$ to 2.8 (95\% CI, 1.5 to 5.4 ) to $9.4(95 \% \mathrm{CI}, 4.7$ to 18.9$)$ in the subclinical, micro-, and macroalbuminuria groups, respectively. Similar increases were seen by strata of eGFR: the adjusted hazard for AKI was 3.5 (95\% CI, 1.7 to 7.3), 12.9 (95\% CI, 5.9 to 28.1 ), and 10.7 (95\% CI, 3.6 to 31.9 ), in participants with a baseline eGFR between 45 and 59, 30 and 44, and 15 and $29 \mathrm{ml} / \mathrm{min}$ per $1.73 \mathrm{~m}^{2}$, respectively.

Interactions between UACR and age, race, gender, and eGFR were tested using a fully adjusted Cox proportional hazards model. The interactions were NS between UACR and gender $(P=0.8)$, UACR and eGFR $(P=0.1)$, and UACR and age $(P=$ $0.08)$; they were statistically significant between UACR and race $(P=0.046)$. All significant and borderline significant interactions were further explored by subgroup analysis. Overall, the trends remained the same. For instance, in fully adjusted analyses using the participants with no albuminuria within the respective race as a reference, the hazard ratio for an AKI event was 1.8 (95\% CI, 1.3 to 2.6 ), 2.2 (95\% CI, 1.5 to 3.4 ), and 4.5 (95\% CI, 2.7 to 7.4 ) in the white participants with subclinical, micro-, and macroalbuminuria, respectively, and 2.2 ( $95 \% \mathrm{CI}, 1.3$ to 3.8$), 2.3$ (95\% CI, 1.4 to 3.9 ), and 6.2 (95\% CI, 3.2 to 12.0 ) in black participants within the same respective UACR categories.

Finally, all analyses were repeated using eGFR calculated by the CKD-EPI equation. The hazard ratios of AKI, adjusted for age, gender, race, cardiovascular risk factors, and categories of UACR, were 1.8 ( $95 \%$ CI, 1.1 to 2.8 ), 1.2 (95\% CI, 0.9 to 1.7 ), 2.0 (95\% CI, 1.4 to 2.8 ), 3.2 ( $95 \%$ CI, 2.1 to 4.7 ), 8.2 (95\% CI, 7.3 to 18.3 ), and 7.0 (95\% CI, 3.2 to 15.0 ) in the categories $\geq 105,75$ to 89,60 to 74,45 to 59,30 to 44 , and 15 to $29 \mathrm{ml} / \mathrm{min}$ per $1.73 \mathrm{~m}^{2}$, respectively, using 90 to $104 \mathrm{ml} / \mathrm{min}$ per $1.73 \mathrm{~m}^{2}$ as

Table 4. Hazard ratio of AKI $(95 \% \mathrm{Cl})$ during follow-up by categories of UACR and eGFR adjusted for age, gender, race, and cardiovascular risk factors ${ }^{a}$

\begin{tabular}{|c|c|c|c|c|c|c|}
\hline \multirow{2}{*}{ eGFR $\left(\mathrm{ml} / \mathrm{min}\right.$ per $\left.1.73 \mathrm{~m}^{2}\right)$} & \multicolumn{6}{|c|}{ Albumin-to-Creatinine Ratio (mg/g) } \\
\hline & $<10$ & 10 to 29 & 30 to 299 & $\geq 300$ & Total & Adjusted \\
\hline$\geq 105$ & $1.2(0.7,2.1)$ & $0.7(0.2,2.2)$ & $1.8(0.7,4.3)$ & $8.6(3.3,22.4)$ & $1.1(0.7,1.6)$ & $1.1(0.7,1.6)$ \\
\hline 75 to 89 & $0.8(0.5,1.3)$ & $2.1(1.1,4.0)$ & $2.4(1.1,4.9)$ & $5.6(2.0,15.8)$ & $0.9(0.7,1.3)$ & $0.9(0.7,1.3)$ \\
\hline 60 to 74 & $1.3(0.9,2.0)$ & $3.4(2.0,5.8)$ & $2.9(1.5,5.7)$ & $9.7(4.7,19.9)$ & $1.5(1.1,2.1)$ & $1.5(1.1,2.1)$ \\
\hline 45 to 59 & $2.6(1.5,4.3)$ & $4.5(2.2,9.2)$ & $6.2(3.4,11.5)$ & $5.8(2.0,16.6)$ & $2.9(2.0,4.2)$ & $2.5(1.7,3.7)$ \\
\hline Total & Reference & $1.9(1.4,2.6)$ & $2.6(1.9,3.5)$ & $8.0(5.6,11.6)$ & - & - \\
\hline Adjusted & Reference & $1.9(1.4,2.6)$ & $2.2(1.6,3.0)$ & $4.8(3.2,7.2)$ & - & - \\
\hline
\end{tabular}

a"Total" refers to adjustment by age, gender, race, and cardiovascular risk factors but not the corresponding column or row category (e.g., not eGFR category for UACR category estimates). "Adjusted" includes adjustment for age, gender, race, cardiovascular risk factors, and corresponding column or row category. 


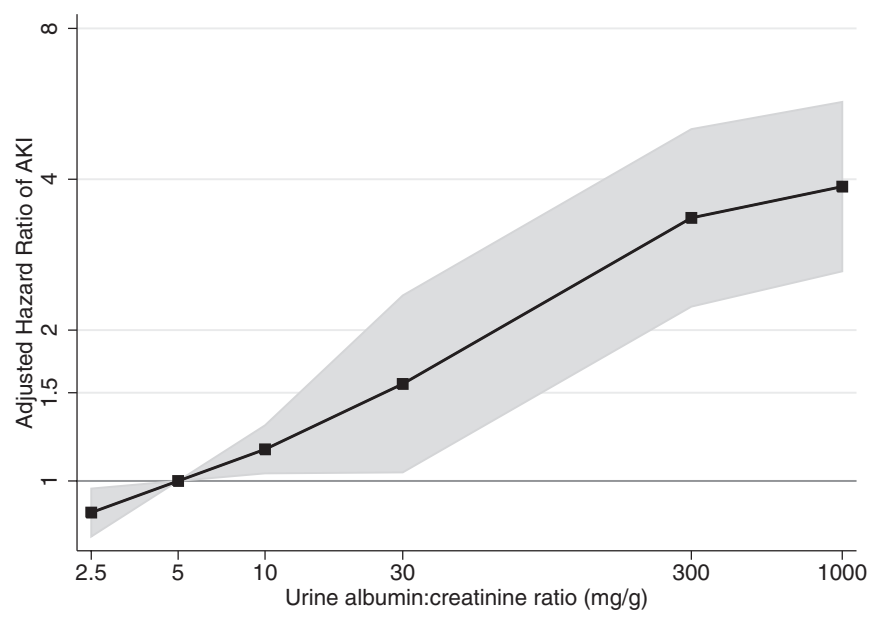

Figure 2. Relative hazard of $A K I$ increases with increasing continuous UACR, adjusted for eGFR, age, gender, race, and cardiovascular risk factors. Linear spline model with a reference UACR of $5 \mathrm{mg} / \mathrm{g}$ and knots at 10, 30, and $300 \mathrm{mg} / \mathrm{g}$ UACR. The shaded area represents $95 \% \mathrm{Cl}$. Cardiovascular risk factors include total cholesterol, presence of diabetes, prevalent cardiovascular disease, smoking status, and measured systolic BP.

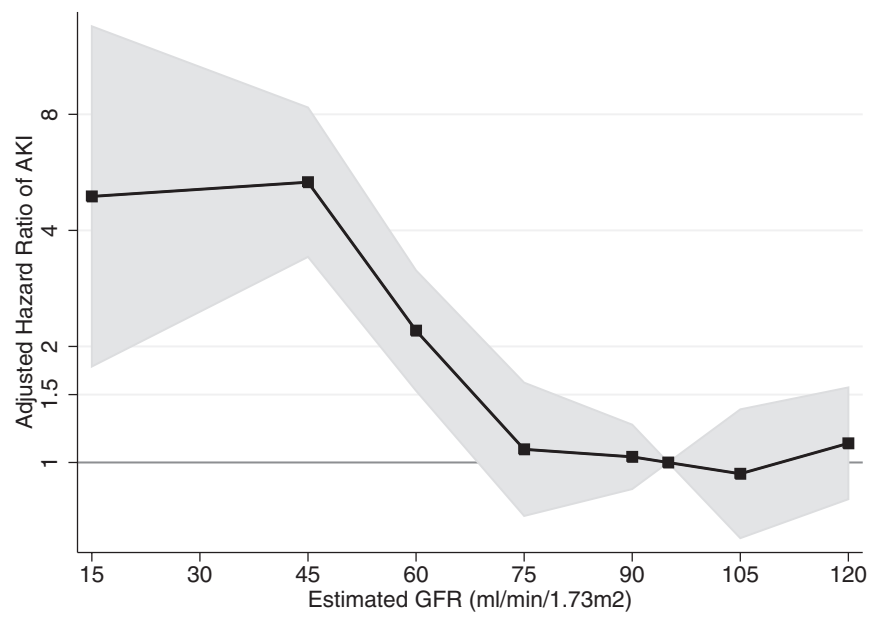

Figure 3. Relative hazard of $A K I$ decreases with increasing continuous eGFR adjusted for UACR, age, gender, race, and cardiovascular risk factors. Linear spline model with a reference eGFR of $95 \mathrm{ml} / \mathrm{min}$ per $1.73 \mathrm{~m}^{2}$ and knots at 45, 60, 75, 90, and 105 $\mathrm{ml} / \mathrm{min}$ per $1.73 \mathrm{~m}^{2}$. The shaded area represents $95 \% \mathrm{Cl}$. Cardiovascular risk factors include total cholesterol, presence of diabetes, prevalent cardiovascular disease, smoking status, and measured systolic BP.

a reference. In all cases, these were larger than the adjusted hazard ratios seen with the corresponding MDRD-calculated eGFR category.

\section{DISCUSSION}

To our knowledge, this is the first population-based study to demonstrate a continuous association between baseline albu- minuria and incidence of AKI, independent of eGFR. We show that levels of albuminuria even below those typically considered pathologic are associated with a higher risk of AKI events, and this risk increases nearly linearly with increasing UACRs. This adds weight to the growing body of evidence that the presence of albuminuria should be considered a high-risk condition and that this risk is in addition to any risk attributable to reduced eGFR. ${ }^{28}$

In our study, baseline characteristics associated with AKI events were consistent with previous reports. ${ }^{11,17-23,25}$ Study participants with an AKI hospitalization or death were more often older, male, and black and more likely to be diabetic, hypertensive, obese, and suffer from CKD and cardiovascular disease. They were also significantly more likely to be on ACEI or ARB therapy. Participants with a known history of AKI were not significantly more likely to experience AKI during our follow-up period; however, there were so few participants fitting this category $(n=20)$ that definitive conclusions cannot be made.

Study participants who experienced AKI were much more likely to die than those who did not, consistent with previous reports. ${ }^{6,7,11,30}$ Not only was in-hospital mortality higher, at $18.9 \%$ versus $2.5 \%$, but follow-up mortality was also higher, with over half of the participants who had an episode of AKI dying during follow-up. As noted by Xue and colleagues, ${ }^{11}$ the crude rate of in-hospital mortality was lower for those participants experiencing hospitalization with AKI as the first discharge diagnosis (thus, theoretically, hospitalized for AKI rather than with AKI). In our study, this is unadjusted for severity of underlying illness or comorbidities; more severe disease and/or more comorbidities likely results in longer hospitalizations and a greater likelihood of AKI and death. If our classification of hospitalizations with versus for AKI is correct, this finding is consistent with reports that much of the excess mortality associated with AKI stems from AKI that is a complication of hospitalization rather than a cause for hospitalization. $^{31,32}$

Our results expand on Hsu and colleagues' findings that the presence of dipstick proteinuria (as a binary measure) is associated with dialysis-requiring AKI. ${ }^{17}$ In their population-based study, baseline dipstick proteinuria (roughly corresponding to $\geq 30 \mathrm{mg}$ per $100 \mathrm{ml}$ of urine) conferred an adjusted odds ratio of dialysis-requiring AKI of 2.79. Here, we have extended this association across the full range of albuminuria and to all AKI hospitalizations, including those that did not require dialytic support. In addition, using a standardized measure of serum creatinine, we estimated the risk of AKI not only for participants with CKD, but also for those with an eGFR in the normal range. Our results indicate that the presence of albuminuria, even at a level below that which would be consistently detected by dipstick, is associated with an increased risk of AKI, even in patients with an eGFR $>60 \mathrm{ml} / \mathrm{min}$ per $1.73 \mathrm{~m}^{2}$.

Furthermore, we demonstrate that the risks of AKI associated with albuminuria and reductions in eGFR are present even after accounting for certain possible confounders, such as 
cardiovascular procedures and ACEI and ARB therapy. Patients with albuminuria and CKD are at increased risk for cardiovascular events. ${ }^{27,33,34}$ Open heart surgery and percutaneous coronary intervention are associated with increased risk of AKI. ${ }^{19-21}$ By censoring the episodes of AKI occurring during these high-risk hospitalizations, we show that the association of AKI with impaired renal function is not mediated solely by increased high-risk operations and procedures. Similarly, patients with albuminuria and CKD are often prescribed ACEI or ARB therapy, which in turn has been linked to increased risk of AKI. ${ }^{35}$ Any association between ACEI and ARB therapy and AKI risk should be interpreted cautiously given the probable confounding by indication (e.g., those with a higher risk of AKI are more likely to be on ACEI or ARB medications); however, our analysis provides evidence that the risk associated with impaired renal function is independent of baseline renin-angiotensin blockade.

There are several unique strengths to our data. First, the cohort was followed prospectively for AKI events. Unlike our cohort, studies in AKI often rely on nephrology consultations to identify cases of AKI ${ }^{36}$ which can introduce selection bias. Second, the baseline measurements of albuminuria and creatinine were undertaken in a standardized manner, and the temporal association between UACR and eGFR measurement and AKI is clear. Third, our study's follow-up is relatively long (mean of 8.0 years) with little attrition, and the active surveillance of hospitalizations likely results in few missed hospitalizations.

The major limitation of our study is the reliance on billing codes for identification of AKI episodes. We used a validated algorithm ${ }^{37}$ with an estimated sensitivity and specificity of $35.4 \%$ and $97.7 \%$, respectively, to identify AKI hospitalizations; however, because the rate of AKI events is relatively low, the positive predictive value of ICD-9-coded AKI may be as low as 30\% to $40 \% .{ }^{38}$ Our identification strategy may have missed cases of AKI, which may differ systematically from identified cases (e.g., in terms of disease severity). In addition, we cannot discern whether the increased incidence of AKI over time reflects trends in disease coding patterns ("code creep"), ${ }^{39}$ misclassification of progressive $\mathrm{CKD}$, an aging population with increased exposure to AKI precipitants, or simply increasing trends in AKI. Future studies are needed to examine the association of albuminuria and eGFR with AKI events identified by a laboratory-based method, such as change in serum creatinine.

Given our reliance on ICD codes, any interpretation of our classification of hospitalizations for AKI versus with AKI is fraught with potential bias given the imperfect translation of clinical situations to medical charts to diagnosis codes. Interpretations of these results should be measured given our uncertainty in classification. Finally, because of random variation in serum creatinine and UACR (e.g., the variation in UACR with respect to time of day), ${ }^{40}$ and the fact that we rely on only one baseline measure of UACR and serum creatinine, our analysis likely underestimates the true association between renal function and AKI risk.
In conclusion, our data from a large, prospectively followed population-based cohort demonstrate a strong and graded association between higher UACR and AKI events. This effect is independent of eGFR, which also exhibits a strong and graded association with incidence of AKI. Patients with even low levels of albuminuria should be considered at increased risk for the development of AKI. As therapy for AKI prevention is refined, preventative measures extended to high-risk groups (e.g.) older persons and those with more advanced CKD) should also be considered for those with albuminuria. ${ }^{41-46}$

\section{CONCISE METHODS}

\section{Data Sources and Study Population}

Data were collected via the ARIC study, a prospective, populationbased cohort of black and white individuals between the ages of 45 and 64 years from four U.S. sites (Forsyth County, NC; Jackson, MS; suburban Minneapolis, MN; and Washington County, MD). The original sample size was 15,792 men and women, identified by probability sampling techniques. Initial enrollment began between 1987 and 1989. Follow-up clinical examinations occurred at approximately 3-year intervals, and individuals participated in annual follow-up telephone interviews. Hospitalizations were captured prospectively from the study inception through December 31, 2005.

Because urine was first assessed at the fourth study visit, our analyses were limited to those who were alive and not lost to follow-up at that time (1996 to 1998), a population with a slightly higher percentage of whites and women than the original cohort. ${ }^{47} \mathrm{~A}$ total of 11,216 participants had complete data at the fourth visit, including UACR, serum creatinine, and cardiovascular risk factors. Of these, 16 had an eGFR $<15 \mathrm{ml} / \mathrm{min}$ per $1.73 \mathrm{~m}^{2}$ (CKD stage 5 ) and were excluded from subsequent analyses.

\section{Data Measurement}

Serum creatinine was measured using a modified kinetic Jaffe method and subsequently corrected for interlaboratory differences by the addition of $0.18 \mathrm{mg} / \mathrm{dl}$ to calibrate to the Cleveland Clinic standard. Standard creatinine was then obtained by multiplying by 0.95 . eGFR was calculated using this standard value in the four-variable MDRD and CKD-EPI equations. ${ }^{48,49}$ Urinary albumin excretion was measured as the ratio of albumin to creatinine (UACR, in milligrams per gram). As reported previously, ${ }^{50}$ samples were collected in a standardized fashion, and quality assurance analysis on 516 of these samples showed a correlation coefficient of 0.95 .

Diabetes was classified as a single fasting serum glucose of $\geq 126$ $\mathrm{mg} / \mathrm{dl}$ or the use of antidiabetic medication; hypertension was defined as a systolic blood pressure (BP) $\geq 140 \mathrm{mmHg}$ and/or diastolic BP $\geq 90 \mathrm{mmHg}$ and/or the use of antihypertensive drugs. ACEI and ARB therapy was determined at the baseline visit by review of all medications taken within the previous 2 weeks.

Vital status and intervening hospitalizations were abstracted during the annual phone interviews, which had a response rate of $96 \%$ in study year 10 and $92 \%$ in study year 20 . In addition, study personnel prospectively tracked discharges at community hospitals and ab- 
stracted the corresponding discharge diagnosis codes (up to 26 discrete codes, length of stay, and in-hospital mortality) for ARIC participants. Hospitalizations outside of the catchment area were ascertained during the follow-up telephone interviews, and discharge records from these outside hospitals were requested and successfully obtained in $>95 \%$ of cases. Between annual interviews, incident deaths were tracked via regular review of local newspaper obituaries, state death lists, community hospital discharge lists, and death certificates from the Department of Vital Statistics.

\section{Data Definitions}

AKI was defined as the presence of one of the following labels in the diagnosis coding: International Classification of Diseases, 9th Revision, Clinical Modification (ICD-9-CM) codes 584.5 to 584.9 or 10th Revision, Clinical Modification (ICD-10-CM) codes N17.0 to 17.9. Our definition of AKI encompassed patients who were hospitalized with AKI, by abstracting the candidate ICD codes from the listed discharge diagnoses, as well as those who died with AKI, by abstracting ICD codes from listed causes of death on the death certificate. Patients were classified as hospitalized with AKI if the discharge diagnosis contained an AKI-defining code or if the patient died during hospitalization and the associated death certificate listed AKI as a cause of death. Patients were classified as hospitalized for AKI if the first discharge diagnosis contained an AKI-defining code. We identified the subset of patients with AKI who received dialysis during their hospitalization using the following ICD-9 and ICD-10 codes: 39.95, 38.95, V39.95, V45.1, V56.0, V56.1, Z49.0, Z49.1, and Z99.2. ${ }^{37}$ High-risk hospitalizations were defined to be any hospitalization with a billing code for open heart surgery or percutaneous coronary intervention.

\section{Data Analysis}

Baseline characteristics of the sample population and hospitalization characteristics were compared using $\chi^{2}$ and $t$ tests. The association of albuminuria and eGFR with AKI hospitalization was analyzed using Cox proportional hazards regression, expressing albuminuria and eGFR as categorical and continuous variables and adjusted for age, gender, race, and cardiovascular risk factors (total cholesterol, diabetes, prevalent cardiovascular disease, current smoking, and systolic BP). A linear spline approach was used to model the continuous relationship between albuminuria and risk of AKI (adjusted for eGFR) and eGFR and risk of AKI (adjusted for albuminuria). For sensitivity analysis, all adjusted hazard ratios were calculated with the inclusion of an interaction variable with UACR to assess for effect modification by age, eGFR, race, and gender. Variables that were noted to have a significant interaction with UACR were then stratified for subgroup analysis. All analyses were performed using Stata 11.0/MP (StataCorp, College Station, TX) and SAS version 9.2 (SAS Institute, Inc., Cary, NC).

\section{ACKNOWLEDGMENTS}

The ARIC study is carried out as a collaborative study supported by National Heart, Lung, and Blood Institute contracts N01-HC-55015,
N01-HC-55016, N01-HC-55018, N01-HC-55019, N01-HC-55020, N01-HC-55021, and N01-HC-55022. We thank the staff and participants of the ARIC study for their important contributions.

\section{DISCLOSURES}

Dr. Lori Bash is currently employed by Merck and Company, Inc. The remaining authors have no relevant competing financial interest to declare.

\section{REFERENCES}

1. Hou SH, Bushinsky DA, Wish JB, Cohen JJ, Harrington JT: Hospitalacquired renal insufficiency: A prospective study. Am J Med 74: 243-248, 1983

2. Shusterman N, Strom BL, Murray TG, Morrison G, West SL, Maislin G: Risk factors and outcome of hospital-acquired acute renal failure. Clinical Epidemiologic Study. Am J Med 83: 65-71, 1987

3. Liano F, Pascual J: Epidemiology of acute renal failure: A prospective, multicenter, community-based study. Madrid Acute Renal Failure Study Group. Kidney Int 50: 811-818, 1996

4. Levy EM, Viscoli CM, Horwitz RI: The effect of acute renal failure on mortality. A cohort analysis. JAMA 275: 1489-1494, 1996

5. Barrantes F, Feng Y, Ivanov $O$, Yalamanchili HB, Patel J, Buenafe $X$, Cheng V, Dijeh S, Amoateng-Adjepong Y, Manthous CA: Acute kidney injury predicts outcomes of non-critically ill patients. Mayo Clin Proc 84: 410-416, 2009

6. Chertow GM, Burdick E, Honour M, Bonventre JV, Bates DW: Acute kidney injury, mortality, length of stay, and costs in hospitalized patients. J Am Soc Nephrol 16: 3365-3370, 2005

7. Thakar CV, Christianson A, Freyberg R, Almenoff P, Render ML: Incidence and outcomes of acute kidney injury in intensive care units: A Veterans Administration study. Crit Care Med 37: 25522558, 2009

8. Hsu CY, Chertow GM, McCulloch CE, Fan D, Ordonez JD, Go AS: Nonrecovery of kidney function and death after acute on chronic renal failure. Clin J Am Soc Nephrol 4: 891-898, 2009

9. Ishani A, Xue JL, Himmelfarb J, Eggers PW, Kimmel PL, Molitoris BA, Collins AJ: Acute kidney injury increases risk of ESRD among elderly. J Am Soc Nephrol 20: 223-228, 2009

10. Lo LJ, Go AS, Chertow GM, McCulloch CE, Fan D, Ordonez JD, Hsu $\mathrm{CY}$ : Dialysis-requiring acute renal failure increases the risk of progressive chronic kidney disease. Kidney Int 76: 893-899, 2009

11. Xue JL, Daniels F, Star RA, Kimmel PL, Eggers PW, Molitoris BA, Himmelfarb J, Collins AJ: Incidence and mortality of acute renal failure in Medicare beneficiaries, 1992 to 2001. J Am Soc Nephrol 17: 11351142,2006

12. Waikar SS, Curhan GC, Wald R, McCarthy EP, Chertow GM: Declining mortality in patients with acute renal failure, 1988 to 2002. J Am Soc Nephrol 17: 1143-1150, 2006

13. Hsu CY, McCulloch CE, Fan D, Ordonez JD, Chertow GM, Go AS: Community-based incidence of acute renal failure. Kidney Int 72 : 208-212, 2007

14. U.S. Renal Data System: USRDS 2009 Annual Data Report: Atlas of Chronic Kidney Disease. Bethesda, MD, National Institute of Diabetes and Digestive and Kidney Diseases, National Institutes of Health, 2009

15. Waikar SS, Liu KD, Chertow GM: Diagnosis, epidemiology and outcomes of acute kidney injury. Clin J Am Soc Nephrol 3: 844-861, 2008

16. Liano F, Junco E, Pascual J, Madero R, Verde E: The spectrum of acute renal failure in the intensive care unit compared with that seen in other 
settings. The Madrid Acute Renal Failure Study Group. Kidney Int Suppl 66: S16-S24, 1998

17. Hsu CY, Ordonez JD, Chertow GM, Fan D, McCulloch CE, Go AS: The risk of acute renal failure in patients with chronic kidney disease. Kidney Int 74: 101-107, 2008

18. Mittalhenkle A, Stehman-Breen CO, Shlipak MG, Fried LF, Katz R, Young BA, Seliger S, Gillen D, Newman AB, Psaty BM, Siscovick D: Cardiovascular risk factors and incident acute renal failure in older adults: The Cardiovascular Health Study. Clin J Am Soc Nephrol 3: 450-456, 2008

19. Mehran R, Aymong ED, Nikolsky E, Lasic Z, lakovou I, Fahy M, Mintz GS, Lansky AJ, Moses JW, Stone GW, Leon MB, Dangas G: A simple risk score for prediction of contrast-induced nephropathy after percutaneous coronary intervention: Development and initial validation. J Am Coll Cardiol 44: 1393-1399, 2004

20. Rihal CS, Textor SC, Grill DE, Berger PB, Ting HH, Best PJ, Singh M, Bell MR, Barsness GW, Mathew V, Garratt KN, Holmes DR Jr: Incidence and prognostic importance of acute renal failure after percutaneous coronary intervention. Circulation 105: 2259-2264, 2002

21. Mehta RH, Grab JD, O'Brien SM, Bridges CR, Gammie JS, Haan CK, Ferguson TB, Petereson ED: Bedside tool for predicting the risk of postoperative dialysis in patients undergoing cardiac surgery. Circulation 114: 2208-2216, 2006

22. Yegenaga I, Hoste E, Van Biesen W, Vanholder R, Benoit D, Kantarci G, Dhondt A, Colardyn F, Lameire N: Clinical characteristics of patients developing ARF due to sepsis/systemic inflammatory response syndrome: Results of a prospective study. Am J Kidney Dis 43: 817824,2004

23. Godet G, Fleron MH, Vicaut E, Zubicki A, Bertrand M, Riou B, Kieffer E, Coriat P: Risk factors for acute postoperative renal failure in thoracic or thoracoabdominal aortic surgery: A prospective study. Anesth Analg 85: 1227-1232, 1997

24. Pascual J, Liano F, Ortuno J: The elderly patient with acute renal failure. J Am Soc Nephrol 6: 144-153, 1995

25. Nash K, Hafeez A, Hou S: Hospital-acquired renal insufficiency. Am J Kidney Dis 39: 930-936, 2002

26. Ninomiya T, Perkovic V, de Galan BE, Zoungas S, Pillai A, Jardine M, Patel A, Cass A, Neal B, Poulter N, Mogensen CE, Cooper M, Marre M, Williams B, Hamet P, Mancia G, Woodward M, MacMahon S, Chalmers J: ADVANCE Collaborative Group: Albuminuria and kidney function independently predict cardiovascular and renal outcomes in diabetes. J Am Soc Nephrol 20: 1813-1821, 2009

27. Foster MC, Hwang SJ, Larson MG, Parikh NI, Meigs JB, Vasan RS, Wang TJ, Levy D, Fox CS: Cross-classification of microalbuminuria and reduced glomerular filtration rate: Associations between cardiovascular disease risk factors and clinical outcomes. Arch Intern Med 167: 1386-1392, 2007

28. Hallan SI, Ritz E, Lydersen S, Romundstad S, Kvenild K, Orth SR: Combining GFR and albuminuria to classify CKD improves prediction of ESRD. J Am Soc Nephrol 20: 1069-1077, 2009

29. Jorgensen L, Jenssen T, Heuch I, Jacobsen BK: The combined effect of albuminuria and inflammation on all-cause and cardiovascular mortality in nondiabetic persons. J Intern Med 264: 493-501, 2008

30. Coca SG, Yusuf B, Shlipak MG, Garg AX, Parikh CR: Long-term risk of mortality and other adverse outcomes after acute kidney injury: A systematic review and meta-analysis. Am J Kidney Dis 53: 961-973, 2009

31. Feest TG, Round A, Hamad S: Incidence of severe acute renal failure in adults: Results of a community based study. BMJ 306: 481-483, 1993

32. Kaufman J, Dhakal M, Patel B, Hamburger R: Community-acquired acute renal failure. Am J Kidney Dis 17: 191-198, 1991

33. Gerstein HC, Mann JF, Yi Q, Zinman B, Dinneen SF, Hoogwerf B, Hallé JP, Young J, Rashkow A, Joyce C, Nawaz S, Yusuf S: HOPE Study Investigators. Albuminuria and risk of cardiovascular events, death, and heart failure in diabetic and nondiabetic individuals. JAMA 286: 421-426, 2001
34. Valmadrid CT, Klein R, Moss SE, Klein BE: The risk of cardiovascular disease mortality associated with microalbuminuria and gross proteinuria in persons with older-onset diabetes mellitus. Arch Intern Med 160: 1093-1100, 2000

35. Arora P, Rajagopalam S, Ranjan R, Kolli H, Singh M, Venuto R, Lohr J: Preoperative use of angiotensin-converting enzyme inhibitors/angiotensin receptor blockers is associated with increased risk for acute kidney injury after cardiovascular surgery. Clin J Am Soc Nephrol 3: 1266-1273, 2008

36. Mehta RL, Pascual MT, Soroko S, Savage BR, Himmelfarb J, Ikizler TA, Paganini EP, Chertow GM; Program to Improve Care in Acute Renal Disease: Spectrum of acute renal failure in the intensive care unit: The PICARD experience. Kidney Int 66: 1613-1621, 2004

37. Waikar SS, Wald R, Chertow GM, Curhan GC, Winkelmayer WC, Liangos O, Sosa MA, Jaber BL: Validity of International Classification of Diseases, Ninth Revision, Clinical Modification codes for acute renal failure. J Am Soc Nephrol 17: 1688-1694, 2006

38. Garg AX, Parikh CR: Yin and yang: Acute kidney injury and chronic kidney disease. J Am Soc Nephrol 20: 8-10, 2009

39. Seiber EE: Physician code creep: Evidence in Medicaid and state employee health insurance billing. Health Care Financ Rev 28: 83-93, 2007

40. Miller WG, Bruns DE, Hortin GL, Sandberg S, Aakre KM, McQueen MJ, Itoh Y, Lieske JC, Seccombe DW, Jones G, Bunk DM, Curhan GC, Narva AS; National Kidney Disease Education Program-IFCC Working Group on Standardization of Albumin in Urine: Current issues in measurement and reporting of urinary albumin excretion. Clin Chem 55 24-38, 2009

41. Weisbord SD, Palevsky PM: Prevention of contrast-induced nephropathy with volume expansion. Clin J Am Soc Nephrol 3: 273 280, 2008

42. Benko A, Fraser-Hill M, Magner P, Capusten B, Barrett B, Myers A, Owen RJ; Canadian Association of Radiologists: Canadian Association of Radiologists: Consensus guidelines for the prevention of contrastinduced nephropathy. Can Assoc Radiol J 58: 79-87, 2007

43. Kagan A, Sheikh-Hamad D: Contrast-induced kidney injury: Focus on modifiable risk factors and prophylactic strategies. Clin Cardiol 33: 62-66, 2010

44. Kelly AM, Dwamena B, Cronin P, Bernstein SJ, Carlos RC: Metaanalysis: Effectiveness of drugs for preventing contrast-induced nephropathy. Ann Intern Med 148: 284-294, 2008

45. Navaneethan SD, Singh S, Appasamy S, Wing RE, Sehgal AR: Sodium bicarbonate therapy for prevention of contrast-induced nephropathy: A systematic review and meta-analysis. Am J Kidney Dis 53: 617-627, 2009

46. Pannu N, Wiebe N, Tonelli M; Alberta Kidney Disease Network: Prophylaxis strategies for contrast-induced nephropathy. JAMA 295: 2765-2779, 2006

47. Bash LD, Astor BC, Coresh J: Risk of incident ESRD: A comprehensive look at cardiovascular risk factors and 17 years of follow-up in the Atherosclerosis Risk in Communities (ARIC) study. Am J Kidney Dis 55: 31-41, 2010

48. Levey AS, Coresh J, Greene T, Marsh J, Stevens LA, Kusek JW, Van Lente F; Chronic Kidney Disease Epidemiology Collaboration: Expressing the modification of diet in renal disease study equation for estimating glomerular filtration rate with standardized serum creatinine values. Clin Chem 53: 766-772, 2007

49. Levey AS, Stevens LA, Schmid CH, Zhang YL, Castro AF III, Feldman HI, Kusek JW, Eggers P, Van Lente F, Greene T, Coresh J: CKD-EPI (Chronic Kidney Disease Epidemiology Collaboration). A new equation to estimate glomerular filtration rate. Ann Intern Med 150: 604 612, 2009

50. Hsu CC, Brancati FL, Astor BC, Kao WH, Steffes MW, Folsom AR, Coresh J: Blood pressure, atherosclerosis, and albuminuria in 10,113 participants in the atherosclerosis risk in communities study. $J$ Hypertens 27: 397-409, 2009 